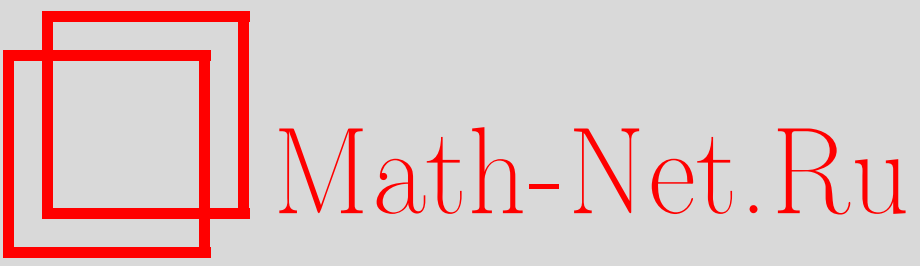

С. В. Знаменский, Е. А. Знаменская, Существование аналитических первообразных на произвольном подмножестве комплексной плоскости, УМH, 2000, том 55, выпуск 1, 183-184

DOI: https://doi.org/10.4213/rm264

Использование Общероссийского математического портала Math-Net.Ru подразумевает, что вы прочитали и согласны с пользовательским соглашением

http: //www . mathnet.ru/rus/agreement

Параметры загрузки:

IP : 54.174 .149 .18

26 апреля 2023 г., 14:48:14 


\title{
СУЩЕСТВОВАНИЕ АНАЛИТИЧЕСКИХ ПЕРВООБРАЗНЫХ НА ПРОИЗВОЛЬНОМ ПОДМНОЖЕСТВЕ КОМПЛЕКСНОЙ ПЛОСКОСТИ
}

\author{
С. В. ЗНАМЕНСКИЙ, Е. А. ЗНАМЕНСКАЯ
}

Хорошо известно [1; с. 100], [2], что существование голоморфных первообразных в области $D$ эквивалентно ее односвязности. Поэтому на открытом множестве голоморфная первообразная существует тогда и только тогда, когда каждая его связная компонента односвязна. В общем случае даже выпуклости всех компонент недостаточно для существования голоморфной первообразной (пример 1$)$.

Пусть $M$ - произвольное множество комплексного пространства, $\mathscr{U}(M)$ - совокупность всех окрестностей множества $M$, т.е. открытых множеств, содержащих $M$. Семейство $\mathscr{U}(M)$ частично упорядочено и направлено по включению, т.е. для любого конечного набора $U_{1}, \ldots, U_{k}$ окрестностей существует меньшая окрестность (не обязательно входящая в этот набор). Голоморфность (аналитичность) на множестве $M$ понимается в смысле ростков [3]. Это означает, что элементом пространства $H(M)$ является росток, т.е. класс эквивалентности в множестве пар вида (окрестность, голоморфная в ней функция) по следующему отношению: две пары $\left(U^{\prime}, f^{\prime}\right)$ и $\left(U^{\prime \prime}, f^{\prime \prime}\right)$ эквивалентны, если существует меньшая окрестность $U \subset \mathscr{U}(M)$, в которой эти функции голоморфны и совпадают. Для ростков операции сложения и умножения на число получаются применением соответствующих операций к функциям из произвольных представителей классов эквивалентности.

Обозначим $H(M)$ пространство функций, аналитических на множестве $M$.

ПримеР 1. Функция $f(z)=\sum_{k=0}^{\infty} 2^{-k}\left(z-(2 k+1)^{-1}\right)^{-1}$ не имеет первообразной на односвязном множестве $M=\{-i\} \cup(i, 1+i] \cup\left(\bigcup_{k=1}^{\infty}\left[(2 k)^{-1}-i,(2 k)^{-1}+i\right]\right)$.

Допустим, что существует $y \in H(M): y^{\prime}=f$. Так как точка $-i \in M$, то при достаточно большом $k$ функция $y$ голоморфна на отрезке $\left[(2 k+2)^{-1}-i,(2 k)^{-1}-i\right]$. Рассмотрим границу $\gamma$ прямоугольника с вершинами в точках $(2 k+2)^{-1}+i,(2 k)^{-1}+i,(2 k)^{-1}-i,(2 k+2)^{-1}-i$. В силу сделанного предположения о существовании первообразной $\int_{\gamma} f(z) d z=0$, но $\int_{\gamma} f(z) d z=$ $2 \pi i 2^{-k} \neq 0$, где $z_{k}=(2 k+1)^{-1}$ - простой полюс функции $f(z)$, попадаюший внутрь кривой $\gamma$. Следовательно, для выбранной функции $f(z)$ первообразной в $M$ не существует.

Теорема 1. Для множества $M \subset \mathbb{C}$ следующие условия әквивалентны:

(1) для любой функиии $f \in H(M)$ существует $y \in H(M)$, для которой $y^{\prime}=f$;

(2) каждая окрестность множества $M$ содержит его односвязную окрестность;

(3) каждое замкнутое подмножество $S \in \overline{\mathbb{C}} \backslash M$ содержится в некотором континууме $K \in \overline{\mathbb{C}} \backslash M$.

ДокАЗАТЕЛЬСтво. $(1) \Rightarrow(2)$ Пусть $U \supset M$ - произвољная окрестность. Чтобы показать, что она содержит односвязную подокрестность, заметим, что множество $\mathbb{C} \backslash U$ сепарабельно как подпространство сепарабельного пространства $\mathbb{C}[4 ;$ с.56, утв. 3.10 .9$]$ и потому содержит всюду плотную последовательность $\left\{z_{k}\right\}_{k=1}^{\infty}$. Положим $f(z)=\sum_{k=0}^{\infty} 2^{-k}\left(z-z_{k}\right)^{-1}$. Согласно условию (1) существует $y \in H(V): y^{\prime}=f, U \supset V \supset M$. Добавим к $V$ все точки пересечения множества $U$ с ограниченными связными компонентами множества $\mathbb{C} \backslash V$. Если таким образом полученное открытое множество $V$ неодносвязно, то в нем по определению существует замкнутая кривая $\gamma$, обходящая хотя бы одну точку $\zeta$ ограниченной связной компоненты множества $\mathbb{C} \backslash V$. По построению $V$ точка $\zeta$ не лежит в $U$.

Обозначим через $D$ односвязную область, ограниченную кривой $\gamma$. Так как точки последовательности $\left\{z_{k}\right\}_{k=1}^{\infty}$ всюду плотны в $\mathbb{C} \backslash U$, то в некоторой окрестности точки $\zeta$ существует хотя бы одна точка $z_{k}$ последовательности, принадлежащая $D$. Но тогда $\int_{\gamma} f(z) d z=2 \pi i \sum_{z_{k}} \in D^{2} \neq 0$.

$(2) \Rightarrow(1)$ Справедливость импликации следует из определения аналитической на множестве функции и существования голоморфной первообразной в односвязной области.

Работа выполнена при поддержке Российского фонда фундаментальных исследований (грант № 99-01-00951). 
(2) $\Rightarrow(3)$ Пусть $S \subset \overline{\mathbb{C}} \backslash M$ замкнуто. Из условия (2) следует существование односвязной окрестности $V \subset \mathbb{C} \backslash S$ множества $M$. Тогда $K=\mathbb{C} \backslash V$ - искомый континуум.

$(3) \Rightarrow(2)$ Рассмотрим произвольную окрестность $U \supset M$. Согласно условию (3) существует континуум $K \subset \mathbb{C} \backslash M$, содержащий множество $\mathbb{C} \backslash U$. Тогда $V=\mathbb{C} \backslash K$-требуемая односвязная окрестность множества $M$.

Для полноты рассмотрения исследуем теперь уравнение или систему линейных диффференциальных уравнений конечного порядка с постоянными коэффициентами $A y=f$, где

$$
A=\left(\begin{array}{cccc}
L_{11} & L_{12} & \ldots & L_{1 n} \\
\vdots & & & \vdots \\
L_{m 1} & L_{m 2} & \ldots & L_{m n}
\end{array}\right), \quad L_{i j}=\sum_{k=1}^{p_{i j}} a_{i j k} \frac{d^{k}}{d z^{k}}, \quad y=\left(\begin{array}{c}
y_{1} \\
\vdots \\
y_{n}
\end{array}\right), \quad f=\left(\begin{array}{c}
f_{1} \\
\vdots \\
f_{m}
\end{array}\right) .
$$

Для краткости будем говорить, что система $A y=f$ разрешима на множестве $M \subset \mathbb{C}$, если для любых функций $f_{1}, \ldots, f_{m} \in H(M)$ в $H(M)$ существуют такие функции $y_{1}, \ldots, y_{n}$, что $A y=f$.

Теорема 2. Для любой матрищы вида А справедлива одна из следующих альтернаmuв:

(1) все миноры порядка $m$ матрицы $A$ взаимнопрость, при этом существует непрерывный правый обратный $\kappa$ л линейный дифференциальный оператор с постоянными коэффиичентами, решающий систему $A y=f$,

(2) все миноры порядка $m$ матрицы $A$ тождественно равны нулю $($ или $n<m)$, при этом система $A y=f$ неразрешима ни на одном множестве $M \subset \mathbb{C}$,

(3) множество общих нулей миноров порядка $m$ матрицы А конечно, при этом система $A y=f$ разрешима на тех и только тех множествах, на которых для любой функции существует первообразная.

ДоказАтЕЛьСтво. С помощью элементарных преобразований матрищу $A$ можно [5; с. 162] привести к диагональной матрице $A^{\prime}$. Известно [5; с. 155], что эквивалентные квадратные матрицы имеют один и тот же наибольший общий делитель миноров одинакового порядка. Доказательство этого утверждения без существенных изменений распространяется на случай прямоугольных матриц.

Предположим, что матрица $A^{\prime}$ содержит хотя бы одну нулевую строку (если хотя бы один из диагональных элементов нулевой, то в этом случае все миноры $m$-го порядка матрицы $A$ тождественно равны нулю, в противном случае выполняется неравенство $n<m$ ), тогда в матрице $A$ есть линейно зависимые строки. Пусть $k$-я строка матрицы $A$ является линейной комбинацией строк с номерами $k_{1}, k_{2}, \ldots, k_{s}$. Для функций $f_{1}, \ldots, f_{m}$, где $f_{k}$ не равна соответствующей линейной комбинации функций $f_{k_{1}}, \ldots, f_{k_{s}}$, решения не существует ни на одном множестве.

Пусть теперь матрица $A^{\prime}$ не содержит ни одной нулевой строки. Если произведение диагональных элементов есть многочлен нулевой степени (т.е. наибольший общий делитель миноров порядка $m$ матрицы $A$ - постоянное число), то матрица $A$ имеет правую обратную матрицу и соответствующий ей дифференциальный оператор решает систему $A y=f$ для любой правой части.

В оставшемся случае произведение диагональных элементов - многочлен ненулевой степени, а поэтому имеет конечное число нулей. Разрешимость первоначальной системы определяется разрешимостью уравнений $p_{i}\left(\frac{d}{d z}\right) y_{i}=f_{i}, i=1, \ldots, m$.

Разложение многочлена $p_{i}\left(\frac{d}{d z}\right)$ на линейные множители сводит решение уравнения к решению уравнений первого порядка, которые экспоненциальной заменой приводятся к уравнению $y^{\prime}=f$, полностью исследованному в теореме 1 .

\section{СПИСОК ЛИТЕРАТУРЫ}

[1] Шабат Б. В. Введение в комплексный анализ, ч. 1. М.: Наука, 1985. [2] Знаменский С. В. // Матем. заметки. 1989. Т. 45. № 1. С. 16-19. [3] . № 2. Р. 77-95. [4] Дьедонне ЖК. Основы современного анализа. М.: Мир, 1964. [5] Мальцев А. И. Основы линейной алгебры. М.: Наука, 1970.

Институт программных систем РАН, г. Переславль-Залесский

Принято редколлегией

E-mail: znamensk@rustex.botik.ru, ekaterin@rustex.botik.ru

22.12 .1999 